\title{
WEAK* COMPACT CONVEX SETS \\ WITH SEPARABLE EXTREMAL SUBSETS HAVE THE RADON-NIKODÝM PROPERTY
}

\author{
RICHARD D. BOURGIN
}

\begin{abstract}
The result stated in the title, as well as several other related results, is derived.
\end{abstract}

In this note we present a natural generalization of a well-known result of Dunford and Pettis. At the original writing our results prompted us to conjecture a still stronger theorem which we communicated to, and which has subsequently been settled by, S. Fitzpatrick. Continued justification for this note stems from the simplicity of both statements and proofs contained herewith.

Recall that a bounded subset $D$ of a Banach space is said to be $s$-dentable if for each $\varepsilon>0$ there corresponds a point $x_{\varepsilon}$ in $D$ which cannot be written as a (possibly infinite) convex combination of points in $D$ each of distance at least $\varepsilon$ from $x_{\varepsilon} ; D$ is subset $s$-dentable if each of its subsets is $s$-dentable. The notational convention employed throughout is that $A$ denotes a weak* compact and norm separable subset of a dual Banach space $Y^{*}$. Several corollaries, including that mentioned in the title, follow from Theorem 1.

THEOREM 1. $A$ is subset $s$-dentable.

Two demonstrations of this assertion are indicated. The first uses a theorem of Haydon to reduce the problem to a well-known topological argument of Namioka, while the second is by martingales. It will be convenient to denote by $B_{\varepsilon}(x)\left(\bar{B}_{\varepsilon}(x)\right)$ the open (closed) $\varepsilon$-ball centered at a point $x$.

Proof 1. According to Haydon's theorem [5, Theorem 3.3] a weak* compact convex set $K$ in a dual space $X^{*}$ is the norm closed convex hull of its extreme points whenever $\operatorname{ex}(K)$ (the set of extreme points of $K$ ) is norm separable. As Haydon's original argument is quite complicated we follow the ideas of J. Bourgain's elementary one [1] here. Thus suppose $K \subset X^{*}$ is weak* compact and convex and that $\left\{x_{i}^{*}\right\}$ is a norm dense subset of $\operatorname{ex}(K)$. Pick $\varepsilon>0$ and $x^{*} \in K$. According to the theorem of Choquet, Bishop, and DeLeeuw (see, for example, [10, p. 24]) there is a probability measure $\mu$ on $K$

Received by the editors January 2, 1977 and, in revised form, May 30, 1977.

AMS (MOS) subject classifications (1970). Primary 46B99.

Key words and phrases. Weak* compact norm separable sets, subset s-dentability, RadonNikodým property. 
with barycenter $x^{*}$ such that $\mu\left(\cup_{i=1}^{\infty} B_{\varepsilon}\left(x_{i}^{*}\right) \cap K\right)=1$ (since this latter set is a weak $F_{\sigma}$ set containing $\left.\operatorname{ex}(K)\right)$. Let $C_{n}=\bar{B}_{\varepsilon}\left(x_{n}^{*}\right) \backslash \cup_{i=1}^{n-1} \bar{B}_{\varepsilon}\left(x_{i}^{*}\right)$. If $\mu\left(C_{n}\right)=$ 0 let $\mu_{n}$ be the point mass at $x_{n}^{*}$; otherwise let $\mu_{n}$ be the normalized restriction of $\mu$ to $C_{n}\left(\mu_{n}=\left.\mu\left(C_{n}\right)^{-1} \mu\right|_{C_{n}}\right)$. Evidently the barycenter $y_{n}^{*}$ of $\mu_{n}$ lies in $\bar{B}_{\varepsilon}\left(x_{n}^{*}\right) \cap K$, and the sequence $\left\{\left[\sum_{i=1}^{n} \mu\left(C_{i}\right)\right]^{-1} \sum_{i=1}^{n} \mu\left(C_{i}\right) y_{i}^{*}\right\}_{n=1}^{\infty}$ converges in norm to $x^{*}$. Because the point $\left[\sum_{i=1}^{n} \mu\left(C_{i}\right)\right]^{-1} \sum_{i=1}^{n} \mu\left(C_{i}\right) x_{i}^{*}$ is within $\varepsilon$ of the $n$th term of this sequence, convex combinations of the $x_{i}^{*}$ are norm dense in $K$. Consequently, $K$ is norm separable and is the norm closed convex hull of its extreme points.

Let $B \subset A$ be any subset and take $K=$ weak* $^{*}$ closed convex hull $(B)$. By the Milman theorem [10, p. 9] $\operatorname{ex}(K) \subset$ weak* $^{*} \operatorname{closure}(B) \subset A$ so that $\operatorname{ex}(K)$ is norm separable and, hence, by Haydon's theorem, $K$ itself is weak* compact, convex, and norm separable. By Namioka's result [9, Theorem 2.2] the set of points of $\operatorname{ex}(K)$ at which the identity map $i:\left(K\right.$, weak $\left.{ }^{*}\right) \rightarrow$ ( $K$, norm) is continuous is a weak ${ }^{*}$ dense $G_{\delta}$ subset of $\operatorname{ex}(K)$. If $y^{*} \in \operatorname{ex}(K)$ is such a point of continuity, appropriate application of the Milman theorem and separation theorem shows that there are a point $x_{0} \in Y$ and $\alpha \in \mathbf{R}$ so that $y^{*} \in\left\{x^{*} \in K: x^{*}\left(x_{0}\right)>\alpha\right\}$, and this weak* slice of $K$ has norm diameter less than $\varepsilon$. The Milman theorem applied again shows that this slice contains points of $B$. Hence $B$ is $s$-dentable, as was to be shown.

Proof 2. In [7, Theorem 3.1] Maynard essentially made the following construction: Suppose that a bounded set $D$ in a Banach space $X$ is not $s$-dentable. Then there is an $X$-valued martingale $\left(f_{n}, \Sigma_{n}\right)$ based on $([0,1), \Sigma, \lambda)(\Sigma=$ Lebesgue measurable sets in $[0,1) ; \lambda=$ Lebesgue measure $)$ such that

(a) $\cup_{n=1}^{\infty}$ Range $f_{n}$ is a countable set in $D$, and

(b) there is an $\varepsilon>0$ such that $\left\|f_{n+1}(t)-f_{n}(t)\right\| \geqslant \varepsilon$ for each $t$ in $[0,1)$, $n=1,2, \ldots$.

If $A$ is not subset $s$-dentable then construct a 'Maynard martingale' as above with values in a non-s-dentable subset $B$ of $A$. For technical reasons, replace $Y$ (if it is not separable) by an appropriate separable subspace $Z$ of $Y$ so that the restriction map $T: A \rightarrow Z^{*}$ is an affine isometry and a weak* homeomorphism. Henceforth assume, then, that $\left\{y_{k}\right\}_{k=1}^{\infty}$ is norm dense in the unit sphere of $Y$. For each $F \in Y^{* *}$ the classical martingale convergence theorem [3, Theorem 4.1, p. 319] asserts the pointwise convergence of the bounded real-valued martingale $\left(F \circ f_{n}, \Sigma_{n}\right)$, except perhaps on an exceptional set $M_{F}$ with $\lambda\left(M_{F}\right)=0$. If for some $t$ the sequence $\left\{f_{n}(t)\right\}_{n=1}^{\infty}$ is not weak* convergent, then there is a $y_{k}$ such that $\left\{f_{n}(t)\left(y_{k}\right)\right\}_{n=1}^{\infty}$ is not convergent. It follows that weak* $\lim f_{n}(t) \equiv f(t)$ exists, except perhaps on a subset $M$ of $\cup_{k=1}^{\infty} M_{y_{k}}$, a set of Lebesgue measure 0 . Pick any $a^{*} \in A$ and define $f(t)=a^{*}$ on $M$.

We next indicate why $f$ is Bochner integrable. First of all, $f$ is separably valued. Next, $f$ is weakly $\Sigma$-measurable. Indeed, for $y \in Y$ the map $f(\cdot)(y)$ is $\Sigma$-measurable since it is the almost sure pointwise limit of the $f_{n}(\cdot)(y)$. Hence $f^{-1}(H)$ is in $\Sigma$ for each weak* open half space $H \subset Y^{*}$. Observe that each 
norm open subset $A$ is a countable union of weak* compact subsets of $A$ (balls intersected with $A$, in fact) so that the weak* and norm Borel subsets of $A$ coincide. Moreover, each weak* open subset of $A$ is the intersection with $A$ of a countable union of finite intersections of weak ${ }^{*}$ open half spaces. Putting these facts together we conclude that $f^{-1}(0) \in \Sigma$ for each relatively norm open subset 0 of $A$. Since $F^{-1}(V) \cap A$ is relatively norm open in $A$ when $F \in Y^{* *}$ and $V$ is open in $\mathbf{R}$, we have $(F \circ f)^{-1}(V)=f^{-1}\left(F^{-1}(V) \cap A\right) \in$ $\Sigma$. Thus $f$ is weakly $\Sigma$-measurable. Since Range $f \subset A$, a bounded set, $\int\|f\| d \lambda<\infty$ so that $f$ is Bochner integrable [6, Theorem 7.5.11].

The conditional expectation of $f$ given $\Sigma_{n}$ is $f_{n}$, since for $W \in \Sigma_{n}$ and $y \in W$ we have

$$
\left(\int_{W} f d \lambda\right)(y)=\int_{W} f(t)(y) d \lambda(t)=\int_{W} f_{n}(t)(y) d \lambda(t)=\left(\int f_{n} d \lambda\right)(y) .
$$

Consequently, $\int_{W} f d \lambda=\int_{W} f_{n}, d \lambda$, whence $\mathbf{E}\left[f \mid \Sigma_{n}\right]=f_{n}$. Lévy's theorem [8, Theorem 13, p. 30] on the continuity of the conditional expectation operator applies (even when the range is a Banach space [8, Remark 14c, p. 31]) and asserts that $f$ is the almost sure pointwise limit of the $f_{n}$ 's. Since this is in conflict with the choice of the $f_{n}$ 's (see condition (b)) the proof is complete.

Professor I. Namioka, to whom we communicated Theorem 1 (with Proof 2) has kindly supplied us with Proof 1 .

A closed bounded convex set $K \subset X$ has the Radon-Nikodym Property (RNP), if for every probability space $(\Omega, \mathcal{G}, P)$ and $X$-valued measure $m$ on $\mathcal{G}$ which is absolutely continuous with respect to $P$, and whose average range $\{m(G) / P(G): G \in \mathcal{G}, P(G)>0\}$ is contained in $K$, there is a Bochner integrable function $f: \Omega \rightarrow K$ for which $m(G)=\int_{G} f d P$ for each $G \in \mathcal{G}$. The Banach space $X$ has the (RNP) if each of its closed bounded convex subsets has the (RNP). A modification of Maynard's results in [7] shows that $K$ (closed bounded and convex in $X$ ) has the (RNP) if and only if it is subset $s$-dentable. (See, for example, [2]). The theorem of Dunford and Pettis [4] follows easily.

COROLlaRY 2. Separable dual spaces have the (RNP).

Proof. Since each closed ball is weak* compact and norm separable, Theorem 1 applies. The result follows from Maynard's characterization of spaces with the (RNP).

CoROllaRY 3. If $X^{*}$ lacks the (RNP) there is a bounded countable set in $X^{*}$ whose weak* closure is not norm separable.

Proof. Pick a bounded countable set in $X^{*}$ which is not $s$-dentable. (This is possible by virtue of Maynard's theorem, together with his observation that each bounded non-s-dentable set contains a countable non-s-dentable subset [7, Lemma 2.2].) The weak* closure of such a set cannot be norm separable by Theorem 1 .

Corollary 3 was first proved by C. Stegall [11, Corollary 5] who also showed that the converse need not hold. 
Corollary 4. Let $K$ be a weak* compact convex set in $X^{*}$ for which $\operatorname{ex}(K)$ is a norm separable set. Then $K$ has the (RNP).

Proof. It follows from Haydon's theorem (see Proof 1 of Theorem 1) that $K$ is norm separable. Hence Theorem 1, followed by the localized Maynard result (quoted before Corollary 3 ), applies.

Based on Theorem 1, as well as indirect corroborating evidence, we advanced the following conjecture in an earlier version of this paper. Let $K$ be a weak* compact convex set in a dual Banach space and suppose that $K$ has the (RNP). Then $K$ is the weak* closed convex hull of its weak* strongly exposed points. (Recall that $x_{0}^{*} \in K$ is a weak* strongly exposed point if there is a point $y \in Y$ such that $x_{0}^{*} \in\left\{x^{*} \in K: x^{*}(y)>M(y)-\alpha\right\}$ and the diameter of these sets tends to 0 as $\alpha \rightarrow 0^{+}$. Here $M(y)=\sup \left\{x^{*}(y)\right.$ : $\left.x^{*} \in K\right\}$.) In this interim, Mr. S. Fitzpatrick has settled this conjecture in the affirmative. Of course, this theorem strengthens our Theorem 1. Its proof is somewhat complicated.

Finally, we raise the following natural question: Let $K \subset Y^{*}$ be weak* compact, convex, and norm separable. Does there exist a Banach space $Z$ and map $T: K \rightarrow Z^{*}$ such that $Z^{*}$ is separable and $T$ is an affine weak*-weak* homeomorphism as well as a norm-norm isometry (or at least homeomorphism) onto its range?

\section{BIBLIOGRAPHY}

1. J. Bourgain, Indirect personal communication.

2. R. Bourgin, Geometric aspects of Banach spaces with the Radon-Nikodym property (to appear).

3. J. L. Doob, Stochastic processes, Wiley, New York, 1953.

4. N. Dunford and B. J. Pettis, Linear operations on summable functions, Trans. Amer. Math. Soc. 47 (1940), 323-392.

5. R. Haydon, An extreme point criterion for separability of a dual Banach space, and a new proof of a theorem of Corson, Quart. J. Math. Oxford Ser. 27 (1976), 379-385.

6. E. Hille, Methods in classical and functional analysis, Addison-Wesley, Reading, Mass., 1972.

7. H. B. Maynard, A geometrical characterization of Banach spaces with the Radon-Nikodym property, Trans. Amer. Math. Soc. 185 (1973), 493-500.

8. P. A. Meyer, Martingales and stochastic integrals. I, Lecture Notes in Math., vol. 284, Springer-Verlag, Berlin, 1972.

9. I. Namioka, Neighborhoods of extreme points, Israel J. Math. 5 (1967), 145-152.

10. R. R. Phelps, Lectures on Choquet's theorem, Van Nostrand, Princeton, N. J., 1966.

11. C. Stegall, The Radon-Nikodym property in conjugate Banach spaces Trans. Amer. Math. Soc. 2006 (1975), 213-233.

Department of Mathematics, University of New Mexico, Albuquerque, New Mexico 87131 